\title{
Propiedades PSICOMÉTRICAS de LA ESCALA DE ANSIEDAd SOCIAL DE LIEBOWITZ EN ESTUDIANTES UNIVERSITARIOS PERUANOS
}

\author{
RULmAN ANDREI FRANCO-JIMENEZ \\ https://orcid.org/0000-0001-8648-834X \\ Universidad Nacional San Luis Gonzaga, Ica, Perú \\ Correo electrónico: andrei.francojimenez@gmail.com
}

Recibido: 27 de octubre del 2020 / Aceptado: 12 de noviembre del 2020

doi: https://doi.org/10.26439/persona2020.n023(2).4903

\begin{abstract}
Resumen. El objetivo del estudio fue analizar las propiedades psicométricas de la Escala de Ansiedad Social de Liebowitz (LSAS), por lo que se evaluó su estructura factorial y su consistencia interna. El estudio estuvo conformado por una muestra de 549 estudiantes de una universidad pública peruana. En el análisis factorial confirmatorio se comparó soluciones de dos, tres, cuatro, cinco y seis factores, evidenciando un mejor ajuste en el modelo de cinco dimensiones propuesto por Caballo, Salazar, Arias, Hofmann y Curtiss (2019), $\chi^{2}=319.43, \mathrm{CFI}=.991, \mathrm{RMSEA}=.043, \mathrm{TLI}=.990, \mathrm{SRMR}=.051$. Asimismo, se analizó la consistencia interna de los cinco factores empleando el coeficiente omega y se obtuvieron valores entre .81 a .62. En conclusión, la LSAS mostró adecuadas propiedades psicométricas en cuanto a los procedimientos evaluados y los resultados se encuentran a favor de una estructura de cinco factores.
\end{abstract}

Palabras clave: trastorno de ansiedad social / Escala de Ansiedad Social de Liebowitz / propiedades psicométricas / análisis factorial / confiabilidad

\section{PSYCHOMETRIC PROPERTIES OF THE LIEBOWITZ SOCIAL ANXIETY SCALE IN PERUVIAN UNIVERSITY STUDENTS}

\begin{abstract}
The aim of the study was to examine the psychometric properties of the Liebowitz Social Anxiety Scale (LSAS) by analyzing its factor structure and internal consistency. The study population consisted of a sample of 549 undergraduate students of a Peruvian public university. A confirmatory factor analysis was used to compare two-, three-, four-, five- and six-factor models. The best model fit indices were found in the five-factor model proposed by Caballo, Salazar, Arias, Hofmann \&
\end{abstract}


Curtiss (2019): $\chi^{2}=319.43, \mathrm{CFI}=0.991, \mathrm{RMSEA}=0.043, \mathrm{TLI}=0.990, \mathrm{SRMR}=0.051$. Also, the internal consistency of the five factors was analyzed by using the omega coefficient, which ranged from 0.81 to 0.62 . In conclusion, LSAS showed appropriate psychometric properties regarding the assessed procedures, and the results support the five-factor structure.

Keywords: social anxiety disorder / Liebowitz Social Anxiety Scale / psychometric properties / factor analysis / reliability 


\section{INTRODUCCIÓN}

El trastorno de ansiedad social (TAS) o fobia social está caracterizado por una ansiedad intensa y persistente en una o más situaciones sociales en las que el individuo está expuesto. En muchos casos, las personas con TAS evitan esta clase de situaciones, lo que hace que el trastorno suela ir acompañado de problemas en el aspecto social (American Psychiatric Association, 2013).

El TAS se encuentra entre los trastornos de ansiedad más frecuentes (Bandelow y Michaelis, 2015), con menor probabilidad de recuperación en ausencia de tratamiento (Bruce et al., 2005) y representa un problema de salud pública en países de Latinoamérica, Norteamérica, África, Europa y Oceanía (Stein et al., 2017), así como en China (Guo et al., 2016) y Japón (Sado et al., 2013). Asimismo, ha sido asociado con una mayor tendencia a presentar síntomas depresivos (Ratnani et al., 2017; Stein et al., 2001), baja autoestima (Maldonado et al., 2013), bajas habilidades sociales (Caballo, Salazar, Irurtia, Olivares y Olivares, 2014) y ausentismo laboral (Alonso et al., 2004).

Pese a su alta prevalencia, el trastorno es raramente diagnosticado (Filho et al., 2010), ya que los síntomas pueden llegar a confundirse con características personales. Por esta razón, son necesarios instrumentos que puedan ayudar en su detección y diagnóstico. La Escala de Ansiedad Social de Liebowitz (LSAS) es el primer instrumento psicométrico desarrollado específicamente para la evaluación de este trastorno (Liebowitz, 1987), y se convirtió rápidamente en uno de los más empleados. Cabe resaltar que la escala fue inicialmente propuesta como una entrevista clínica, aunque posteriormente fue considerado su uso como autoinforme (Baker, Heinrichs, Kim y Hofmann, 2002; Fresco et al., 2001).

Las propiedades psicométricas de la LSAS han sido analizadas y se ha encontrado una excelente consistencia interna y validez convergente (Heimberg et al., 1999), así como una buena confiabilidad test-retest (Baker et al., 2002). En cuanto a la validez discriminante del instrumento, diversos estudios han mostrado que sujetos con TAS presentan puntajes significativamente mayores que quienes no tienen el trastorno (Bobes, Badía, García y Dal-Ré, 1999; Heimberg et al., 1999; Heimberg y Holaway, 2007; Oakman, Van Ameringen, Mancini y Farvolden, 2002). Asimismo, la LSAS ha sido utilizada para establecer la validez convergente de otros instrumentos tales como el Social Phobia Inventory (SPIN) (Connor et al., 2000), la Social Interaction Anxiety Scale (SIAS) y la Social Phobia Scale (SPS) (De Beurs, Tielen y Wollmann, 2014), así como el Cuestionario de Ansiedad Social para Adultos (CASO) (Salazar, Caballo y Arias, 2016).

No obstante, uno de los puntos más controvertidos en los estudios psicométricos del instrumento es lo que respecta a su estructura factorial. Distintas investigaciones han encontrado una cantidad diferente de factores: tres (Levin, Marom, Gur, Wechter y 
Hermesh, 2002), cuatro (Bobes et al., 1999; Safren et al., 1999; Sugawara et al., 2012), cinco (Caballo, Salazar, Arias, Hofmann y Curtiss, 2019; Baker et al., 2002; Terra et al., 2006) e incluso seis (Stein, Kasper, Andersen, Nil y Lader, 2004). Estos resultados contradictorios pueden deberse a las diferencias culturales de las muestras empleadas, ya que los síntomas del TAS pueden verse influenciados por el contexto cultural (Hofmann, Asnaani y Hinton, 2010).

Por estas razones, se hace necesario evaluar las propiedades psicométricas de la LSAS en un contexto peruano. El objetivo del presente estudio es determinar la estructura factorial que mantenga un mejor ajuste considerando factores propuestos por distintos autores. Asimismo, analizar la consistencia interna del instrumento.

\section{MÉTODO}

\section{Participantes}

La muestra consistió en 549 estudiantes de pregrado reclutados en 11 facultades distintas de una universidad estatal peruana. El total estuvo conformado por 261 mujeres (47,5\%) y 288 varones (52,5\%), con edades que van entre 16 y 43 años ( $M=21,29$; $D E=5,23)$. Se utilizó un muestreo accidental, ya que la investigación se conformó por los sujetos disponibles a los que se tuvo acceso (Kerlinger y Lee, 2002). Todos los participantes dieron previamente su consentimiento informado para el estudio.

\section{Instrumento}

La Escala de Ansiedad Social de Liebowitz (LSAS) es un instrumento que cuenta con 24 ítems referidos a miedo/ansiedad y evitación en situaciones que suelen ser problemáticas para pacientes con fobia social. Cada uno de los ítems evalúa ambas variables. Los evaluados miden su miedo/ansiedad en una escala de Likert de 4 grados que varía de 0 (ninguna ansiedad) a 3 (bastante ansiedad). Asimismo, evalúan su grado de evitación en una escala de Likert de 4 grados que varía de 0 (ninguna evitación) a 3 (usualmente lo evito). El puntaje total se obtiene al sumar el puntaje de las dos subescalas. En cuanto a las propiedades psicométricas de la versión en español, se encontró una estructura de cuatro factores (González et al., 1998) como también de cinco (Caballo, Salazar, Irurtia, Arias y Nobre-Sandoval, 2013). En el presente estudio se utilizó una versión adaptada al contexto peruano de la LSAS, en la que se halló una excelente consistencia interna mediante el coeficiente alfa de .94 para la escala de ansiedad y de .92 para la escala de evitación (Franco-Jimenez y Pérez, 2018). 


\section{Procedimiento}

Los individuos que participaron en el estudio lo hicieron de forma anónima y voluntaria, después de firmar un consentimiento informado. Asimismo, se les indicó que los datos recolectados serían usados para fines estrictamente académicos.

La escala fue administrada de manera colectiva en las aulas de clase por un grupo de estudiantes de psicología capacitados previamente, en un único encuentro con cada sujeto y con una duración aproximada de 30 minutos.

\section{RESULTADOS}

El análisis psicométrico fue realizado mediante el lenguaje y programa de análisis estadístico $\mathrm{R}$, específicamente, el paquete Lavaan. Se empleó el análisis factorial confirmatorio ( $\mathrm{AFC}$ ), el cual es una técnica estadística multivariada que permite determinar el ajuste entre una estructura factorial propuesta y los datos recolectados. Los modelos analizados en el estudio fueron los de dos, tres (Levin et al., 2002), cuatro (Safren et al., 1999) y cinco factores correlacionados (Caballo et al., 2019). El método de estimación usado fue el de los mínimos cuadrados ponderados con media y varianza ajustada (WLSMV). Los índices de ajuste seleccionados para la interpretación del modelo factorial fueron el índice de aproximación de la raíz de cuadrados medios del error (RMSEA), raíz cuadrada de la media de residuos al cuadrado (SRMR), índice de ajuste comparativo (CFI), índice de Tucker-Lewis (TLI), además del chi cuadrado $\left(\chi^{2}\right)$. Se consideraron como adecuados valores del RMSEA $\leq .06, \mathrm{CFI} / \mathrm{TLI} \geq .90$ y SRMR $\leq .10$ (Hu y Bentler, 1999; Mehmetoglu y Jakobsen, 2016).

En la tabla 1, se observa la matriz de correlaciones policóricas del instrumento, donde el ítem 13, "orinar en servicios públicos", presenta correlaciones menores a .30 con los demás ítems de la LSAS, incluidos aquellos con quienes suele compartir el mismo factor en distintos modelos, tales como el ítem 22, el ítem 24 (Baker et al., 2002; Caballo et al., 2019; Stein et al., 2004) y el ítem 1 (Baker et al., 2002; Safren et al., 1999). 
Rulman Andrei Franco-Jimenez

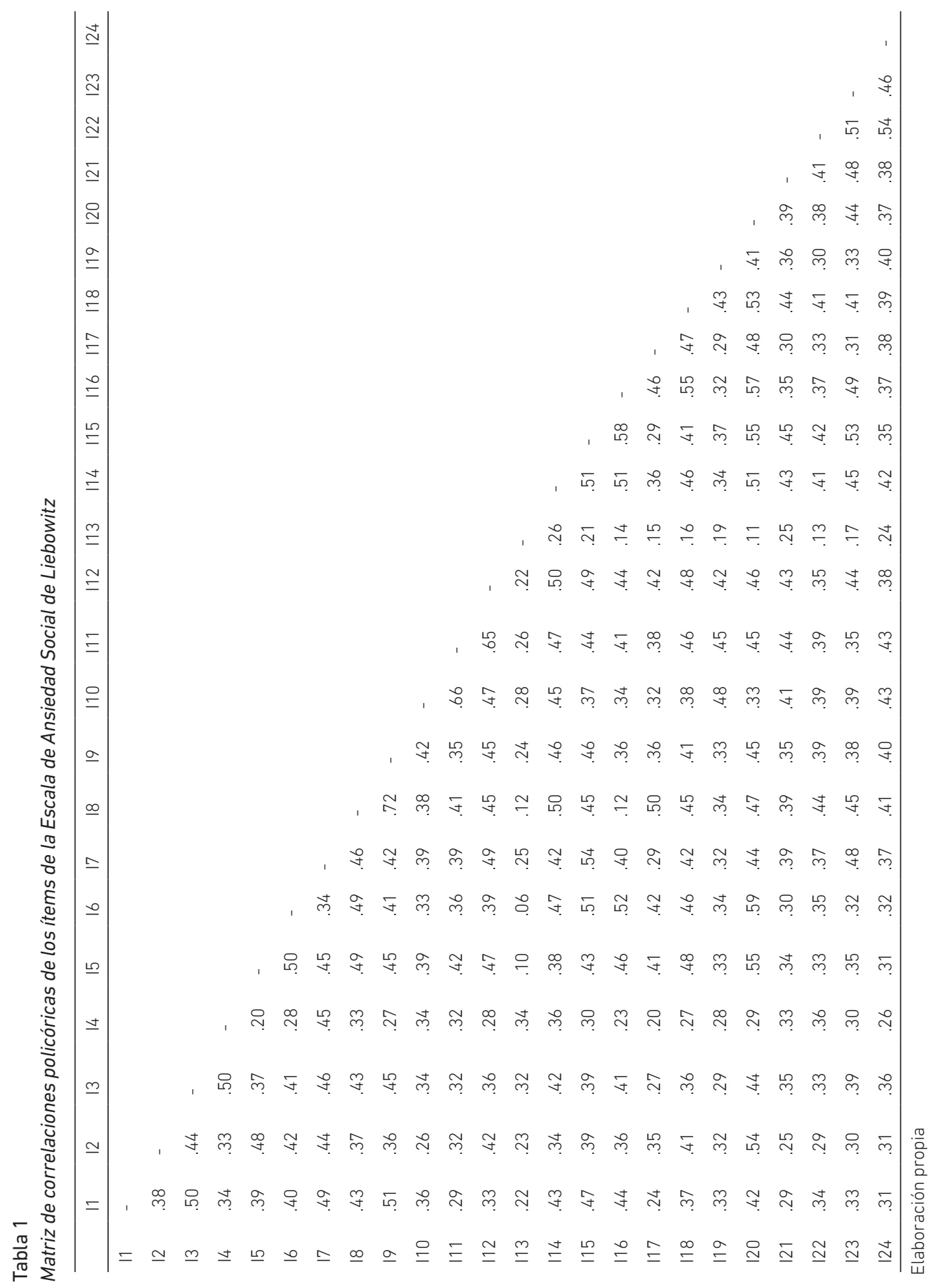


La tabla 2 muestra el resultado del AFC en la escala de ansiedad evidenciándose un ajuste adecuado en todos los modelos, aunque destaca la estructura de cinco factores de Caballo et al. (2019), $\chi^{2}(160, N=549)=319.43, p<.001, \mathrm{CFI}=.991, \mathrm{RMSEA}=.043$, $\mathrm{TLI}=.990, \mathrm{SRMR}=.051$. Cabe resaltar que estos cinco factores son similares a los propuestos por Baker et al. (2002) y por Stein et al. (2004), por lo que se procedió a analizar sus propiedades.

Tabla 2

Índices de ajuste de seis modelos de la Escala de Ansiedad Social de Liebowitz ( $N=549)$

\begin{tabular}{|c|c|c|c|c|c|c|c|}
\hline Modelos & $\chi^{2}$ & $\mathrm{gl}$ & $\mathrm{p}$ & $\begin{array}{l}\text { RMSEA } \\
{[90 \% \text { IC] }}\end{array}$ & $\mathrm{CFI}$ & TLI & SRMR \\
\hline $\begin{array}{l}6 \text { factores: } \\
\text { Stein et al. }\end{array}$ & 517.27 & 327.00 & $<.001$ & $\begin{array}{c}.046 \\
{[.041-.052]}\end{array}$ & .989 & .988 & .055 \\
\hline $\begin{array}{l}5 \text { factores: } \\
\text { Caballo et al. }\end{array}$ & 319.43 & 160.00 & $<.001$ & $\begin{array}{c}.043 \\
{[.036-.049]}\end{array}$ & .991 & .990 & .051 \\
\hline $\begin{array}{l}5 \text { factores: } \\
\text { Baker et al. }\end{array}$ & 451.99 & 199.00 & $<.001$ & $\begin{array}{c}.048 \\
{[.042-.054]}\end{array}$ & .989 & .987 & .055 \\
\hline $\begin{array}{l}4 \text { factores } \\
\text { Safren et al. }\end{array}$ & 377.77 & 146.00 & $<.001$ & $\begin{array}{c}.054 \\
{[.047-.061]}\end{array}$ & .985 & .983 & .059 \\
\hline $\begin{array}{l}3 \text { factores: } \\
\text { Levin et al. }\end{array}$ & 605.07 & 249.00 & $<.001$ & $\begin{array}{c}.051 \\
{[.046-.056]}\end{array}$ & .987 & .985 & .058 \\
\hline $\begin{array}{l}2 \text { factores: } \\
\text { interacciones } \\
\text { sociales y } \\
\text { desempeño }\end{array}$ & 690.82 & 251.00 & $<.001$ & $\begin{array}{c}.057 \\
{[.052-.062]}\end{array}$ & .983 & .982 & .060 \\
\hline
\end{tabular}

Elaboración propia

En la tabla 3 se observan las cargas factoriales del modelo considerado, las cuales se encuentran entre .49 y .82, demostrando ser adecuadas, a excepción del ítem 13 que presenta una carga factorial de 29 .

Tabla 3

Cargas factoriales de la solución estandarizada del análisis factorial confirmatorio del modelo de cinco factores de Caballo et al. (2019)

\begin{tabular}{|c|c|c|c|c|c|}
\hline Ítem & F1 & F2 & F3 & F4 & F5 \\
\hline 5. Hablar con personas que tienen autoridad & .64 & & & & \\
\hline $\begin{array}{l}\text { 6. Actuar, hacer una representación o dar una } \\
\text { charla ante un público }\end{array}$ & .65 & & & & \\
\hline 15. Ser el centro de la atención & .73 & & & & \\
\hline 16. Intervenir en una reunión & .66 & & & & \\
\hline
\end{tabular}


(continuación)

20. Exponer un informe ante un grupo

2. Participar en grupos pequeños

3. Comer en lugares públicos

4. Beber con otras personas en lugares públicos

7. Ir a una fiesta

13. Orinar en servicios públicos

21. Intentar conquistar a alguien

22. Devolver una compra a una tienda

24. Resistir a la presión de un vendedor insistente

8. Trabajar mientras le están observando

9. Escribir mientras le están observando

10. Llamar por teléfono a alguien que no conoce demasiado

11. Hablar con personas que usted no conoce demasiado

12. Conocer gente nueva

19. Mirar a los ojos a alguien que usted no conoce demasiado

23. Dar una fiesta
.71 .55

Elaboración propia

Por otro lado, se muestra en la tabla 4 que las correlaciones entre los cinco factores propuestos por Caballo et al. (2019) en la escala de ansiedad fueron moderadas, variando entre .67 a .88 .

Tabla 4

Correlación entre los cinco factores de la Escala de Ansiedad Social de Liebowitz

\begin{tabular}{llllll}
\hline Factores & 1 & 2 & 3 & 4 & 5 \\
\hline 1. Hablar en público & - & & & & \\
2. Comer/beber delante de los demás & .82 & - & & & \\
3. Comportamientos asertivos & .72 & .78 & - & & \\
4. Trabajar/escribir delante de otras personas & .73 & .67 & .70 & - \\
5. Interactuar con extraños & .79 & .74 & .88 & .67 & - \\
\hline
\end{tabular}

Elaboración propia 
Se utilizó el coeficiente omega (McDonald, 1999) con el objetivo de evaluar la consistencia interna del instrumento. En relación con los cinco factores propuestos por Caballo et al. (2019), los valores fueron moderados, pues se obtuvieron coeficientes de entre .81 a .62 .

\section{DISCUSIÓN}

El objetivo del estudio fue analizar la estructura factorial de la LSAS en una muestra de 549 universitarios peruanos mediante el análisis factorial confirmatorio considerando los modelos de Caballo et al. (2019), Safren et al. (1999), Levin et al. (2002), Baker et al. (2002), Stein et al. (2004) y el planteamiento original de dos factores: interacciones sociales y desempeño (Liebowitz, 1987). Asimismo, determinar la consistencia interna del instrumento mediante el coeficiente omega.

Al emplearse el AFC, todos los modelos evaluados demostraron adecuados índices de ajuste, aunque destacó el modelo de cinco factores propuesto por Caballo et al. (2019), el cual incluye los factores también planteados en estudios previos tales como hablar en público (Safren et al., 1999; Oakman et al., 2002), comer/beber delante de otras personas (Safren et al., 1999; Stein et al., 2004; Oakman et al., 2002; Terra et al., 2006; Baker et al., 2002), conductas asertivas (Stein et al., 2004; Baker et al., 2002), trabajar/escribir mientras se es observado (Safren et al., 1999; Stein et al., 2004; Oakman et al., 2002; Baker et al., 2002) e interacciones con desconocidos (Safren et al., 1999, Oakman et al., 2002). Al evaluar la consistencia interna de los cinco factores mediante el coeficiente omega, se encontraron valores moderados.

Por otro lado, al analizar la matriz de correlaciones policóricas, se observa correlaciones bajas entre el ítem 13, "orinar en servicios públicos", y los otros ítems ubicados en el mismo factor. Además, presenta una baja carga factorial, lo que da soporte a la idea propuesta por Caballo et al. (2019) de que este ítem puede no contribuir a la estructura factorial de la LSAS.

Cabe resaltar que en el modelo factorial de Caballo et al. (2019) solamente dos ítems se encuentran ubicados en el factor "trabajar/escribir mientras se es observado". Resultados similares han sido obtenidos en los modelos de Baker et al. (2002) y Stein et al. (2004), quienes encuentran solo tres ítems en tal factor. Es posible que la LSAS se beneficie de una mayor cantidad de ítems dirigidos a evaluar el temor a ser observado, al ser una dimensión importante en el TAS (Heimberg, Mueller, Holt, Hope y Liebowitz, 1992).

Los resultados deben analizarse en el marco de ciertas limitaciones, por lo que no pueden considerarse definitivos. En primer lugar, el estudio estuvo conformado exclusivamente por una muestra de estudiantes universitarios, de modo que debería tenerse 
precaución al considerar generalizar los resultados a otros grupos socioculturales. Asimismo, se debe realizar investigaciones futuras en grupos de diferentes regiones del Perú. En segundo lugar, una limitación en el uso de autoinformes es la posibilidad de que existan sesgos en las respuestas de los sujetos debido a factores como deseabilidad social.

En resumen, los resultados proporcionan evidencia de que la LSAS presenta una estructura de cinco factores, tal como fue propuesto por Caballo et al. (2019), y demuestra adecuadas propiedades psicométricas en universitarios peruanos.

\section{REFERENCIAS}

Alonso, J., Angermeyer, M. C., Bernert, S., Bruffaerts, R., Brugha, T. S., Bryson H., ... Vollebergh, W. A. (2004). Disability and quality of life impact of mental disorders in Europe: results from the European Study of the Epidemiology of Mental Disorders (ESEMeD) project. Acta Psychiatrica Scandinavica Supplementum, 109(420), 38-46. https://doi.org/10.1111/j.1600-0047.2004.00325.x

American Psychiatric Association. (2013). Diagnostic and Statistical Manual of Mental Disorders (DSM-5). Washington D. C.: Autor.

Baker, S. L., Heinrichs, N., Kim, H. J., y Hofmann, S. G. (2002). The Liebowitz Social Anxiety Scale as a self-report instrument: a preliminary psychometric analysis. Behavior Research and Therapy, 40(6), 701-715. https://doi.org/10.1016/s00057967(01)00060-2

Bandelow, B., y Michaelis, S. (2015). Epidemiology of anxiety disorders in the 21st century. Dialogues in Clinical Neuroscience, 17(3), 327-335. Recuperado de https:// www.dialogues-cns.org/contents-17-3/dialoguesclinneurosci-17-327/

Bobes, J., Badía, X., L. A., García, M., González, M. P., y Dal-Ré, R. (1999). Validación de las versiones en español de los cuestionarios Liebowitz Social Anxiety Scale, Social Anxiety and Distres Scale y Sheehan Disability Inventory para la evaluación de la fobia social. Medicina Clínica, 112(14), 530-538. Recuperado de https:// www.unioviedo.es/psiquiatria/wp-content/uploads/2017/03/1999_Bobes_ Validacion_MedClinicaBCN.pdf

Bruce S. E., Yonkers, K. A., Otto, M. W., Eisen, J. L., Weisberg, R. B., Pagano, M. ... Keller M. B. (2005). Influence of psychiatric comorbidity on recovery and recurrence in generalized anxiety disorder, social phobia, and panic disorder: a 12-year prospective study. The American Journal of Psychiatry, 162(6), 1179-1187. https:// doi.org/10.1176/appi.ajp.162.6.1179 
Caballo, V. E., Salazar, I. C., Arias, V., Hofmann, S. G., y Curtiss, J. (2019). Psychometric properties of the Liebowitz Social Anxiety Scale in a large cross-cultural Spanish and Portuguese speaking sample. Brazilian Journal of Psychiatry, 41(2), 122-130. https://doi.org/10.1590/1516-4446-2018-0006

Caballo, V. E., Salazar, I. C., Irurtia M. J., Arias, B., y Nobre-Sandoval, L. (2013). The assessment of social anxiety through five self-report measures, LSAS-SR, SPAI, SPIN, SPS, and SIAS: a critical analysis of their factor structure. Behavioral Psychology, 21, 423-448. Recuperado de https://www.behavioralpsycho.com/wp-content/ uploads/2019/08/01.Caballo_21-3oa.pdf

Caballo, V. E., Salazar, I. C., Irurtia, M. J., Olivares, P., y Olivares, J. (2014). Relación de las habilidades sociales con la ansiedad social y los estilos/trastornos de la personalidad. Behavioral Psychology, 22(3), 401-422. Recuperado de https://www. behavioralpsycho.com/producto/relacion-de-las-habilidades-sociales-con-laansiedad-social-y-los-estilos-trastornos-de-la-personalidad/

Connor, K. M., Davidson, J. R., Churchill, L. E., Sherwood, A., Foa, E., ... Wisler, R. H. (2000). Psychometric properties of the Social Phobia Inventory (SPIN). New self-rating scale. British Journal of Psychiatry: The Journal of Mental Science, 176, 379-386. https://doi.org/10.1192/bjp.176.4.379

De Beurs, E., Tielen, D., y Wollmann, L. (2014). The Dutch Social Interaction Anxiety Scale and the Social Phobia Scale: reliability, validity, and clinical utility. Psychiatry Journal, 2014, 360193. https://doi.org/10.1155/2014/360193

Filho, A. S., Hetem, L. A. B., Ferrari, M. C. F., Trzesniak, C., Martín-Santos, R., Borduqui, T., ... Crippa, J. A. (2010). Social anxiety disorder: what are we losing with the current diagnostic criteria? Acta Psychiatrica Scandinava, 121(3), 216-226. https:// doi.org/10.1111/j.1600-0447.2009.01459.x

Franco-Jimenez, R. A., y Pérez, C. (2018). Adaptación y validación de la Escala de Ansiedad Social de Liebowitz en muestra clínica de un hospital estatal (tesis de pregrado). Universidad Ricardo Palma, Lima.

Fresco, D. M., Coles, M. E., Heimberg, R. G., Liebowitz, M. R., Hami, S., ... Goetz, D. (2001). The Liebowitz Social Anxiety Scale: a comparison of the psychometric properties of self-report and clinician-administered formats. Psychological Medicine, 31(6), 1025-1036. https://doi.org/10.1017/s0033291701004056

González, M. P., Bobes, J., García, M., Badía, X., Luque, A., y Dal-Ré, R. (1998). Assessing social phobia. The Spanish validation of the "gold standard" clinical scales: the LSAS and the SADS. European Neuropsychopharmacology, 8, S259-S260. https:// doi.org/10.1016/s0924-977x(98)80471-9 
Guo, X., Meng, Z., Huang, G., Fan, J., Zhou, W., Ling, W., ... Su, L. (2016). Meta-analysis of the prevalence of anxiety disorders in mainland China from 2000 to 2015. Scientific Reports, 6, 28033. https://doi.org/10.1038/srep28033

Heimberg, R. G., y Holaway, R. M. (2007). Examination of the know-groups validity of the Liebowitz Social Anxiety Scale. Depression and Anxiety, 454(6), 447-454. https:// doi.org/10.1002/da.20277

Heimberg, R. G., Horner, K. J., Juster, H. R., Safren, S. A., Brown, E. J., Schneier, F. R., ... Liebowitz, M. R. (1999). Psychometric properties of the Liebowitz Social Anxiety Scale. Psychological Medicine, 29(1), 199-212. https://doi.org/10.1017/ s0033291798007879

Heimberg, R. G., Mueller, G. P., Holt, C. S., Hope, D. A., y Liebowitz, M. R. (1992). Assessment of anxiety in social interaction and being observed by others: the Social Interaction Anxiety Scale and the Social Phobia Scale. Behavior Therapy, 23(1), 53-73. https://doi.org/10.1016/S0005-7894(05)80308-9

Hofmann, S. G., Asnaani, M. A., y Hinton, D. E. (2010). Cultural aspects in social anxiety and social anxiety disorder. Depression and Anxiety, 27(12), 1117-1127. https://doi.org 10.1002/da.20759

Hu, L.-t., y Bentler, P. M. (1999). Cutoff criteria for fit indexes in covariance structure analysis: conventional criteria versus new alternatives. Structural Equation Modeling, 6(1), 1-55. https://doi.org/10.1080/10705519909540118

Kerlinger, F. N., y Lee, H. B. (2002). Investigación del comportamiento: métodos de investigación en ciencias sociales. Ciudad de México: McGraw-Hill.

Levin, J. B., Marom, S., Gur, S., Wechter, D., y Hermesh, H. (2002). Psychometric properties and three proposed subscales of a self-report version of the Liebowitz Social Anxiety Scale translated into Hebrew. Depression and Anxiety, 16(4), 143-151. https://doi.org/10.1002/da.10064

Liebowitz, M. R. (1987). Social phobia. Modern Problems in Pharmacopsychiatry, 22, 141-173. https://doi.org/10.1159/000414022

Maldonado, L., Huang, Y., Chen, R., Kasen, S., Cohen, P., y Chen, H. (2013). Impact of early adolescent anxiety disorders on self-esteem development from adolescence to young adulthood. The Journal of Adolescent Health: Official Publication of the Society for Adolescent Medicine, 53(2), 287-292. https://doi.org/10.1016/j. jadohealth.2013.02.025

McDonald, R. P. (1999). Test theory: a unified treatment. Mahwah, NJ: Lawrence Erlbaum Associates, Inc. 
Mehmetoglu, M., y Jakobsen, T. G. (2016). Applied statistics using Stata: a guide for the social sciences. Londres: Sage.

Oakman, J., Van Ameringen, M., Mancini, C., y Farvolden, P. (2002). A confirmatory factor analysis of a self-report version of the Liebowitz Social Anxiety Scale. Journal of Clinical Psychology, 59(1), 149-161. https://doi.org/10.1002/jclp.10124

Ratnani, I. J., Vala, A. U., Panchal, B. N., Tiwari, D. S., Karambelkar, S. S., Sojitra, M. G., y Nagori, N. N. (2017). Association of social anxiety disorder with depression and quality of life among medical undergraduate students. Journal of Family Medicine and Primary Care, 6(2), 243-248. https://doi.org/10.4103/2249-4863.219992

Sado, M., Takechi, S., Inagaki, A., Fujisawa, D., Koreki, A., Mimura, M., y Yoshimura, K. (2013). Cost of anxiety disorders in Japan in 2008: a prevalence-based approach. BMC Psychiatry, 13, 338. https://doi.org/10.1186/1471-244x-13-338

Salazar, I. C., Caballo, V. E., y Arias, B. (2016). Validez de constructo y fiabilidad del "Cuestionario de Ansiedad Social para Adultos" (CASO) en Colombia. Revista Latinoamericana de Psicología, 48(2), 98-107. https://doi.org/10.1016/j. rlp.2015.07.001

Safren, S. A., Heimberg, R. G., Horner, K. J., Juster, H. R., Schneier, F. R., y Liebowitz, M. R. (1999). Factor structure of social fears: the Liebowitz Social Anxiety Scale. Journal of Anxiety Disorders, 13(3), 253-270. https://doi.org/10.1016/s08876185(99)00003-1

Stein M. B., Fuetsch, M., Müller, N., Höfler, M., Lieb, R., y Wittchen, H. (2001). Social anxiety disorder and the risk of depression: a prospective community of adolescents and young adults. Archives of General Psychiatry, 58(3), 251-256. https://doi. org/10.1001/archpsyc.58.3.251

Stein, D. J., Kasper, S., Andersen, E. W., Nil, R., y Lader, M. (2004). Escitalopram in the treatment of social anxiety disorder: analysis of efficacy for different clinical subgroups and symptom dimensions. Depression and Anxiety, 20(4), 175-181. https://doi.org/10.1002/da.20043

Stein, D. J., Lim, C., Roest, A. M., De Jonge, P., Aguilar-Gaxiola, S., Al-Hamzawi, A., ... Scott, K. M. (2017). The cross-national epidemiology of social anxiety disorder: data from the World Mental Health Survey Initiative. BMC Medicine, 15(1), 143. https:// doi.org/10.1017/9781316336168.002

Sugawara, N., Yasui-Furukori, N., Kaneda, A., Sato, Y., Tsuchimine, S., Fujii, A. ... Kaneko, S. (2012). Factor structure of the Liebowitz Social Anxiety Scale in community-dwelling subjects in Japan. Japanese Society of Psychiatry and Neurology, 66(6), 525-528. https://doi.org/10.1111/j.1440-1819.2012.02381.x 
Terra, M. B., Barros, H. M., Stein, A. T., Figueira, I., Athayde, L. D., Gonçalves, M., ... Silveira, D. X. (2006). Consistência interna e estrutura fatorial da versão em português da Escala de Ansiedade Social de Liebowitz entre pacientes alcoolistas. Revista Brasileira de Psiquiatría, 28(4), 265-269. https://doi.org/10.1590/S151644462006005000008 